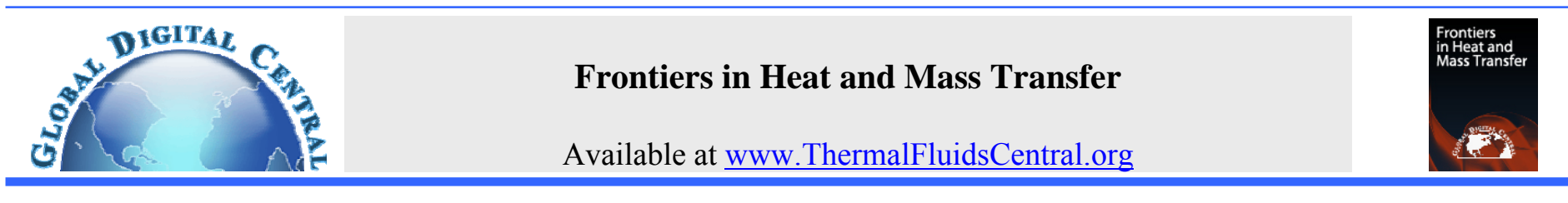

\title{
EFFECTS OF HOMOGENEOUS-HETEROGENEOUS CHEMICAL REACTION AND SLIP VELOCITY ON MHD STAGNATION FLOW OF A MICROPOLAR FLUID OVER A PERMEABLE STRETCHING/SHRINKING SURFACE EMBEDDED IN A POROUS MEDIUM
}

\author{
P. Bala Anki Reddy ${ }^{a,{ }^{*}}$ S. Suneetha ${ }^{b}$ \\ a *Department of Mathematics, Fluid Dynamics division, S.A.S., VIT University, Vellore-632014, India \\ ${ }^{\mathrm{b}}$ Department of Applied Mathematics, Yogi Vemana University, Kadapa-516003, India
}

\begin{abstract}
We report on a mathematical model for analyzing the effects of homogeneous-heterogeneous chemical reaction and slip velocity on the MHD stagnation point flow of electrically conducting micropolar fluid over a stretching/shrinking surface embedded in a porous medium. The governing boundary layer coupled partial differential equations are transformed into a system of non-linear ordinary differential equations, which are solved numerically using the MATLAB bvp4c solver. The effects of physical and fluid parameters such as the stretching parameter, micropolar parameter, permeability parameter, strength of homogeneous and heterogeneous reaction parameter on the velocity and concentration are analyzed, and these results are presented through graphs. The solute concentration at the surface is found to decrease with the strength of the homogeneous reaction, and to increase with heterogeneous reactions, the permeability parameter and stretching or shrinking parameters. Comparison between the previously published results and the present numerical results for various special cases has been done and are found to be an excellent agreement.
\end{abstract}

Keywords: MHD, Stagnation point flow, homogenous-heterogeneous chemical reaction, slip velocity and stretching/shrinking surface.

\section{INTRODUCTION}

Micropolar fluid is a non-Newtonian fluid that belongs to a class of fluids with non-symmetrical stress tensor and is referred to as polar fluid. Micropolar fluids are fluids with internal structures in which coupling between the spin of each particle and the microscope velocity field is taken into account. They represent fluids consisting of rigid, randomly oriented or spherical particles suspended in a viscous medium, where the deformation of fluid particles is ignored. Micropolar fluid theory was introduced by Eringen (1966) in order to describe physical systems, which do not satisfy the Navier-Stokes equations. The equations governing the micro polar fluid involve a spin vector and a micro inertia tensor in addition to the velocity vector. The dynamics of micro polar fluids provides some practical applications, for example turbulent shear flow, the flow of colloidal suspensions, polymeric fluids, liquid crystals, additive suspensions, human and animal blood, analyzing the behaviour of exotic lubricants. The potential importance of micro polar fluids in industrial applications has motivated many researchers to extend the study in numerous ways to include various physical effects. The essence of the theory of micro polar fluid lies in particle suspension (Hudimoto and Tokuoka, 1969), liquid crystals (Lockwood et al., 1987); animal blood (Ariman et al., 1974a), exotic lubricants (Erigen, 1976), etc. An excellent review of the various applications of micro polar fluid mechanics was presented by (Ariman et al.,1974b), Hayat et al., 2016, Ramzan et al., 2016, Sajid et al., (2009a) investigated the exact analytic solution for the three thin film flow problems of a micro polar fluid. The main advantage of using a micro polar fluid model to study the boundary layer flow in comparison with other classes of non-Newtonian fluids is that it takes care of the rotation of the fluid particles by means of an independent kinematic vector called the micro-rotation vector was investigated by Sajid et al., (2009).

The flow of a non-Newtonian fluid over a stretching sheet has attracted considerable attention during the last two decades due to its vast applications in industrial manufacturing such as hot rolling, wire drawing, glass fiber and paper production, drawing of plastic films, polymer extrusion of plastic sheets and manufacturing of polymeric sheets. For the production of glass fiber/plastic sheets, thermo-fluid problem involves significant heat transfer between the sheet and the surrounding fluid. Sheet production process starts solidifying molten polymers as soon as it exits from the slit die. The sheet is then collected by a wind-up roll upon solidification. To improve the mechanical properties of the fiber/plastic sheet we use two ways, the extensibility of the sheet and the rate of cooling. Crane (1970) was the first who reported the analytical solution for the laminar boundary layer flow past a stretching sheet. Several researchers viz. Gupta and Gupta (1977), Dutta et al. (1985), Chen and Char (1988) extended the work of Crane by including the effects of heat and mass transfer under different situations.

Magneto hydrodynamic (MHD) is the science which deals with the motion of highly conducting fluids in the presence of a magnetic field. The motion of the conducting fluid across the magnetic field generates electric currents which change the magnetic field and the action of the

*Corresponding author. Email: pbarmaths@gmail.com 
magnetic field on these currents gives rise to mechanical forces which modify the flow of the fluid. The Magneto hydrodynamic (MHD) character of fluid especially in physiological and industrial processes seems too much important. Such consideration is useful for blood pumping and magnetic resonance imaging (MRI), cancer therapy, hyperthermia etc. Abo-Eldahab and Ghonaim (2003) investigated convective heat transfer in an electrically conducting micropolar fluid at a stretching surface with uniform free stream. Wang et al., (2011) studied with the magnetohydrodynamic flow of a micropolar fluid in a circular cylindrical tube. Eldabe and Ouaf (2006) solved the problem of heat and mass transfer in a hydro magnetic flow of a micropolar fluid past a stretching surface with Ohmic heating and viscous dissipation using the Chebyshev finite difference method.

Hiemenz (1911) first reported the stagnation point flow towards a flat plate. It is worthwhile to note that the stagnation flow appears whenever the flow impinges to any solid object and the local fluid velocity at a point MHD stagnation point flow of a micropolar fluid over a stretching surface with heat source (called the stagnation-point) is zero. Chiam (1994) extended the works of Hiemenz (1911) replaced the solid body a stretching sheet with equal stretching and straining velocities and he was unable to obtain any boundary layer near the sheet. Whereas, Mahapatra and Gupta (2001) reinvestigated the stagnation-point flow towards a stretching sheet considering different stretching and straining velocities and they found two different kinds of boundary layers near the sheet depending on the ratio of the stretching and straining constants. The study of a steady two-dimensional stagnation point flow of a micropolar fluid over a stretching sheet when the sheet was stretched in its own plane and the stretching velocity was proportional to the distance from the stagnation point was examined by Nazar et al. (2004). The resulting coupled equations of nonlinear ordinary differential equations were solved numerically. Hayat et al. (2009a) investigated the two-dimensional Magneto hydrodynamic (MHD) stagnation-point flow of an incompressible micropolar fluid over a nonlinear stretching surface. Hayat et al. (2009b) analyzed the steady two dimensional MHD stagnation point flow of an upper convected Maxwell fluid over the stretching surface. The governing nonlinear partial differential equations were reduced to ordinary ones using the similarity transformation. The homotopy analysis method (HAM) was used to solve these equations. Bhattacharyya (2013) investigated the boundary layer stagnation-point flow of Casson fluid and heat transfer towards a shrinking/stretching sheet. Yacos et al. (2011) have been investigated melting heat transfer in boundary layer stagnation-point flow toward a stretching/shrinking sheet in a micropolar fluid.

The combined heat and mass transfer problems with chemical reactions are of importance in many processes, and therefore have received a considerable amount of attention in recent years. In processes, such as drying, evaporation at the surface of a water body, energy transfer in a wet cooling tower and the flow in a desert cooler, the heat and mass transfer occurs simultaneously. Many chemically reacting systems involve both homogeneous and heterogeneous reactions, with examples occurring in combustion, catalysis, biochemical systems, crops damaging through freezing, cooling towers, fog dispersion, hydrometallurgical processes etc. The interaction between the homogeneous reactions in the bulk of fluid and heterogeneous reactions occurring on some catalytic surfaces is generally very complex, involving the production and consumption of reactant species at different rates both within the fluid and on the catalytic surfaces. A simple mathematical model for homogeneousheterogeneous reactions in stagnation-point boundary-layer flow was initiated by Chaudhary and Merkin (1995(a)). They modeled the homogeneous (bulk) reaction by isothermal cubic kinetics and the heterogeneous (surface) i reaction was assumed to have first-order kinetics. Later Chaudhary and Merkin (1995(b)) extended their previous work to include the effect of loss of the autocatalyst. They studied the numerical solution near the leading edge of a flat plate. A model for isothermal homogeneous-heterogeneous reactions in boundary layer flow of a viscous fluid flow past a flat plate was studied by Merkin (1996). Effects of homogeneous and heterogeneous reactions in flow of nanofluids over a nonlinear stretching surface with variable surface thickness was reported by Hayat et al., (2016) and observed that the homogenous and heterogeneous parameters have opposite behaviors for concentration profile. Ziabakhsh et al. (2010) studied the problem of flow and diffusion of chemically reactive species over a nonlinearly stretching sheet immersed in a porous medium. Chambre and Acrivos (1956) studied an isothermal chemical reaction on a catalytic in a laminar boundary layer flow. They found the actual surface concentration without introducing unnecessary assumptions related to the reaction mechanism. The effect of flow near the twodimensional stagnation point flow on an infinite permeable wall with a homogeneous-heterogeneous reaction was studied by Khan and Pop (2010). They solved the governing nonlinear equations using the implicit finite difference method. It was observed that the mass transfer parameter considerably affects the flow characteristics. Melting and homogeneous/heterogeneous reactions effects in nanofluid flow by a cylinder are addressed by Hayat et al., (2016). It is found that maximum heat transfer and minimum thermal resistance for base fluid suspended multi-wall carbon nanotubes (MWCNTs) when compared with other nanofluids. The behavior of homogeneous parameter $\mathrm{K}$ on concentration profile is sketched for water and kerosene oil base fluids by Hayat et al., (2016). It is analyzed that the concentration field is decreasing function of homogeneous parameter $\mathrm{K}$ for base fluids water and kerosene oil. In fact higher values of homogeneous reaction parameter correspond to larger chemical reaction which consequently reduces the concentration distribution. Hayat et al., (2016) developed numerical analysis for homogeneous-heterogeneous reactions and Newtonian heating in magnetohydrodynamic (MHD) flow of PowellEyring fluid by a stretching cylinder and noticed that the flow accelerates for large values of Powell-Eyring fluid parameter. Hayat et al., (2016) disclose the effects of homogeneous-heterogeneous reactions and melting heat phenomenon in the Magnetohydrodynamic second grade fluid flow. Heat transfer is tackled with heat generation/absorption. Khan and Pop (2012) studied the effects of homogeneous-heterogeneous reactions on the viscoelastic fluid toward a stretching sheet. They observed that the concentration at the surface decreased with an increase in the viscoelastic parameter.

Flow through porous media has various physiological applications such as the flow of blood in the micro-vessels of the lungs which may be treated as a channel bounded by two thin porous layers (Misra and Ghosh (1997)). It is realized that fluid slips at the walls in certain physiological and engineering situations. The no slip boundary condition is a core concept in fluid dynamics in which the fluid and the boundary move with same velocity. Beaver and Joseph (1967) were the first to propose slip boundary condition. The boundary condition proposed by Beaver and Joseph was simplified by Saffman (1971). The existence of slip phenomenon at the boundaries and interfaces has been observed in the flows of rarefied gases, physiological flows, hypersonic flows of chemically reacting binary mixture etc. Also, flows with slip occur for certain problems in chemical engineering, for example, flows through pipes in which chemical reactions occur at the walls, certain two-phase flows and flows in porous slider bearings. Haliza Rosali et al. (2012) studied a micropolar fluid flow towards a permeable stretching or shrinking sheet in a porous medium. Mhd Flow and Heat Transfer through a Porous Medium over a Stretching/Shrinking Surface with Suction was analyzed by F. Ahmad1 et al. (2015). Homogeneousheterogeneous reactions in micropolar fluid flow from a permeable stretching or shrinking sheet in a porous medium was studied by Shaw et al. (2013).

At the macroscopic level, it is well accepted that the boundary condition for a viscous fluid at a solid wall is one of no-slip, i.e., the fluid velocity matches the velocity of the solid boundary. While the noslip condition has been processed experimentally to be accurate for a number of macroscopic flows, it remains an assumption that is not based on physical principles. In many practical applications, the particle 
adjacent to a solid surface no longer takes the velocity of the surface. The particle at the surface has a finite tangential velocity. It slips along the surface. The flow regime is called a slip-flow regime, and this effect cannot be neglected. The study of magneto-micro polar fluid flows in the slip-flow regimes with heat transfer has important engineering applications, e.g., in power generators, refrigeration coils, transmission lines, electric transformers, and heating elements. Mahmoud and Waheed (2010) performed a theoretical analysis to study heat transfer characteristics of magneto hydrodynamic mixed convection flow of a micro polar fluid past a stretching surface with slip. Hayat et al., (2016) presented the effect of Partial slip effect in flow of magnetite $\mathrm{Fe} 3 \mathrm{O} 4$ nanoparticles between rotating stretchable disks. Bakr (2011) analyzed Chemically Reacting Unsteady Magneto hydrodynamic Oscillatory Slip Flow of a Micropolar Fluid in a Planer Channel with Varying Concentration. Hayat et al., (2016) analyzed that larger values of first order slip velocity parameters and magnitude of second order slip velocity parameters correspond to lower velocity. With an increase in slip velocity parameters, stretching velocity is partially transferred to the fluid so velocity profiles decrease. The Effects of Chemical Reaction, Hall, and Ion-Slip Currents on MHD Micropolar Fluid Flow with Thermal were studied by S. S. Motsa1 and S. Shatey (2012). Hayat et al., (2016) studied the MHD three-dimensional flow of nanofluid with velocity slip and nonlinear thermal radiation. Hayat et al., (2016) looks at the influence of an inclined magnetic field on peristaltic transport of hyperbolic tangent nanofluid in inclined channel having flexible walls. Alireza et al. (2013) presented an analytical solution for MHD stagnation point flow and heat transfer over a permeable stretching sheet with chemical reaction.

\section{MATHEMATICAL FORMULATION}

Let us consider the steady two-dimensional stagnation point flow of viscous, incompressible and electrically conducting micropolar fluid over a stretching sheet embedded in a porous medium. The Cartesian coordinate system is used with the $x$-axis along the sheet and the $y$-axis normal to the sheet. Two equal but opposite forces are applied to the stretching sheet so that the surface is stretched, keeping the position of the origin unaltered. A magnetic field $B_{0}$ is applied perpendicular to the sheet. It is assumed that the magnetic Reynolds number is much less than unity so that the induced magnetic field is negligible in comparison to the applied magnetic field. Keeping the origin fixed, it is assumed that the surface is stretched/shrunk with a linear velocity $U W(x)=U_{w} x$, where $U_{W}$ is a constant with $U_{W}>0$ for a stretching sheet, $U_{W}<0$ for a shrinking sheet and $U W=0$ for a static sheet. Also, we consider a simple model for the interaction between a homogeneousheterogeneous reaction involving the two-chemical species $A$ and $B$ in a boundary layer flow proposed by Chaudhary and Merkin (1995a,1995b) of the following form:

$A+2 B \rightarrow 3 B$, Rate $=k_{c} a b^{2}$

$A \rightarrow B$, Rate $=k_{s} a$

Where $a$ and $b$ are concentrations of chemical species $A$ and $B$ respectively, and $k_{c}, k s$ are the constants. It is assumed that the ambient fluid moves with a velocity $u_{e}(x)=U_{\infty} x$, where $U_{\infty}$ is a constant, in

which there is a uniform concentration $a_{0}$ of reactant $A$ and in which there is no auto catalyst $B$ over a flat surface. Under these assumptions and boundary layer approximations the steady two-dimensional stagnation point flow of micropolar fluid towards a stretching sheet embedded in a porous medium is described by the following equations: Continuity Equation

$\frac{\partial u}{\partial x}+\frac{\partial v}{\partial y}=0$ $u \frac{\partial u}{\partial x}+v \frac{\partial u}{\partial y}=u_{e} \frac{d u_{e}}{\partial x}+\left(\frac{\mu+k}{\rho}\right) \frac{\partial^{2} u}{\partial y^{2}}-\frac{k}{\rho} \frac{\partial N}{\partial y}-\frac{\sigma B_{0}^{2}}{\rho}\left(u_{e}-u\right)-\frac{\varepsilon \mu_{e f f}}{\rho k_{1}}\left(u_{e}-u\right)$

Angular Momentum Equation

$u \frac{\partial N}{\partial x}+v \frac{\partial N}{\partial y}=\frac{\gamma}{\rho j} \frac{\partial^{2} N}{\partial y^{2}}-\frac{k}{\rho j}\left(2 N+\frac{\partial u}{\partial y}\right)$

Energy Equation

$u \frac{\partial a}{\partial x}+v \frac{\partial a}{\partial y}=D_{A} \frac{\partial^{2} a}{\partial y^{2}}-k_{c} a b^{2}$

Species Equation

$u \frac{\partial b}{\partial x}+v \frac{\partial b}{\partial y}=D_{A} \frac{\partial^{2} b}{\partial y^{2}}+k_{c} a b^{2}$

where $u$ and $v$ are the velocity components in the $x$ and $y$ directions respectively, $u_{e}$ is the velocity outside the boundary layer, $\mu$ is the dynamic viscosity, $\mu_{e f f}$ is the effective dynamic viscosity, $k$ is the vortex viscosity, $\rho$ is the density of the fluid, $N$ is the micro rotation, $\sigma$ is the electrical conductivity, $B_{0}$ is the uniform magnetic field, $k_{1}$ is the permeability of the porous medium, $j=\frac{v}{U_{\infty}}$ is the micro inertia per unit mass, $\gamma$ is the spin gradient viscosity defined as $\gamma=\mu\left(\frac{1+K_{p}}{2}\right) j, K_{p}=\frac{k}{\mu}$ is the material or micropolar parameter, $D_{A}$ is the diffusive species coefficient of $A$ and $D_{B}$ is the diffusive species coefficient of $B$.

The corresponding boundary conditions are

$$
\begin{aligned}
& u=u_{w}(x)+N v \frac{\partial u}{\partial y}, v=v_{w}, \\
& N=-n \frac{\partial u}{\partial y}, \mathrm{D}_{\mathrm{A}} \frac{\partial a}{\partial y}=k_{s} a, \\
& \mathrm{D}_{B} \frac{\partial b}{\partial y}=k_{s} a \text { at } y=0 \\
& u \rightarrow u_{e}(x), \quad N \rightarrow 0, \quad a \rightarrow a_{0}, b \rightarrow 0 \text { as } y \rightarrow \infty
\end{aligned}
$$

Where $v_{w}$ is the constant mass flux with $v_{w}<0$ for suction and $v_{w}>0$ for injection (blowing) respectively; $N$ is the slip velocity coefficient and $n$ is a constant $(0 \leq n \leq 1)$. Here $n=0$ represents the strong concentration (Guram and Smith, 1980), and $n=1$ represents the turbulent boundary layer flow (Peddieson, 1972). The case $n=1 / 2$ indicates the vanishing of the antisymmetrical part of the stress tensor and denotes weak concentration (Ahmadi, 1976), which is the case considered in the present study.

Now, introducing the following transformation

$\eta=\sqrt{\left(\frac{U_{\infty}}{v}\right)} y, \psi=\sqrt{U_{\infty} v} x f(\eta), N=\sqrt{\left(\frac{U_{\infty}}{v}\right)} U_{\infty} x p(\eta), g(\eta)=\frac{a}{a_{0}}, h(\eta)=\frac{b}{a_{0}}$

Where $\eta$ is the similarity variable and $\psi(x, y)$ is the stream function. The velocity components are defined by $u=\frac{\partial \psi}{\partial y}, v=-\frac{\partial \psi}{\partial x}$.

Substituting (9) into the Equations (4) -(7) and (8), we get the following set of ordinary differential equations

$\left(1+K_{p}\right) f^{\prime \prime \prime}+f f^{\prime \prime}+1-\left(f^{\prime}\right)^{2}+K_{p} p^{\prime}-M\left(1-f^{\prime}\right)+D\left(1-f^{\prime}\right)=0$

$\left(1+\frac{K_{p}}{2}\right) p^{\prime \prime}+f p^{\prime}-f^{\prime} p-K_{p}\left(2 p+f^{\prime \prime}\right)=0$

Linear Momentum Equation 
$\frac{1}{S c} g^{\prime \prime}+f g^{\prime}-k g h^{2}=0$

$\frac{\delta}{S c} h^{\prime \prime}+f h^{\prime}+k g h^{2}=0$

The corresponding boundary conditions are

$f(0)=S, f^{\prime}(0)=\lambda+S_{v} f^{\prime \prime}(0), \mathrm{p}(0)=-n f^{\prime \prime}(0), f^{\prime}(\infty)=1, p(\infty)=0$

$g^{\prime}(0)=K_{s} g(0), \delta h^{\prime}(0)=-K_{s} g(0), g(\infty)=1, h(\infty)=0$

where the primes denote the differentiation with respect to $\eta$, $M=\frac{\sigma B_{0}^{2}}{\rho U_{\infty}}$ is the magnetic parameter, $D=\frac{\mu_{e f f} \varepsilon}{U_{\infty} k_{1} \rho}$ is the permeability parameter, $S c=\frac{v}{D_{A}}$ is the Schmidt number, $K=\frac{k_{c} a_{0}^{2}}{U_{\infty}}$ is a measure of the strength of the homogeneous reaction, $\delta=\frac{D_{B}}{D_{A}}$ is the ratio of the diffusion coefficient, $S=\frac{-v_{w}}{\sqrt{U_{\infty} v}}$ is the suction parameter $(S=0$ represents impermeable, $S>0$ represents suction and $S<0$ represents the injection or blowing), $\lambda=\frac{u_{w}}{u_{e}}$ is the stretching parameter ( $\lambda<0$ represents the shrinking surface, $\lambda>0$ represents the stretching surface and $\lambda=0$ represents the forced convection flow towards the stagnation point on a static surface, $S_{v}=N \sqrt{U_{\infty} v}$ is the slip parameter, $K_{s}=\frac{k_{s} l \mathrm{Re}^{-\frac{1}{2}}}{D_{A}}$ is a measure of the strength of the heterogeneous reaction and $\operatorname{Re}=\frac{U_{\infty} l}{v}$ is the Reynolds number. It is assumed that the diffusion coefficients of chemical species $A$ and $B$ to be of a comparable size. This argument provides us to make further assumption that the diffusion coefficients $\mathrm{D}_{\mathrm{A}}$ and $\mathrm{D}_{\mathrm{B}}$ are equal i.e., $\delta=1$ and thus:

$g(\eta)+h(\eta)=1$

Now Esq. (12) and (13) reduces to

$\frac{1}{S c} g^{\prime \prime}+f g^{\prime}-k g\left(1-g^{2}\right)=0$

With the boundary conditions

$g^{\prime}(0)=K_{s} g(0), g(\infty)=1$.

Also the quantity of physical interest in this problem is the skin friction coefficient which is defined by $C_{f}=\frac{\tau_{w}}{\rho U^{2} / 2}$ where $\tau_{w}=\mu\left(\frac{\partial u}{\partial y}\right)_{y=0}$

Using the similarity variables in Eq. (19), we get

$\operatorname{Re}_{x}^{\frac{1}{2}} C_{f}=(1+K / 2) f^{\prime \prime}(0)$

Where $\operatorname{Re}_{x}=\frac{x U_{e}(x)}{v}$ is the local Reynolds number.

\section{SOLUTION OF THE PROBLEM}

The set of equations (12) to (13) were reduced to a system of first-order differential equations and solved using a MATLAB boundary value problem solver called bvp4c. This program solves boundary value problems for ordinary differential equations of the form $y^{\prime}=f(x, y, p), a \leq x \leq b$, by implementing a collocation method subject to general nonlinear, two-point boundary conditions $g(y(a), y(b), p)$. Here $p$ is a vector of unknown parameters. Boundary value problems (BVPs) arise in most diverse forms. Just about any BVP can be formulated for solution with bvp4c. The first step is to write the $O D E s$ as a system of first order ordinary differential equations. The details of the solution method are presented in Shampine and Kierzenka (2000).

\section{RESULTS AND DISCUSSIONS}

The numerical computations have been carried out using the MATLAB bvp4c solver for several values of the physical parameters arised in the study then acquired results are presented in graphs.

The variations of the velocity and concentration profiles are plotted as a function of $\eta$ for some values of $\lambda$ in Figures 1 and 2 for $K_{p}=0.1, \chi$ $=0.1, S c=1, K=1, s=0.5, n=0.5, \quad K s=1$. In Fig.1, (i) for $\lambda>0$ (stretching surface), the fluid velocity is becoming increasingly greater than the free stream. In this case the fluid velocity decreases with the value of $\eta$ and converges at unity as per the condition. (ii) For $\lambda=0$ (static surface), the fluid velocity initially is stationary, but with $\eta$ value it increases in a non-linear way. (iii) For $\lambda<0$ (shrinking surface), the fluid velocity is initially negative, but it increases with $\eta$, and after a certain value of $\eta$, it becomes positive. For the concentration profile in figure 2 , all the curves are started from the origin and they increase nonlinearly with $\eta$ to follow ' $S$ ' shape and finally reach unity according to the given condition.

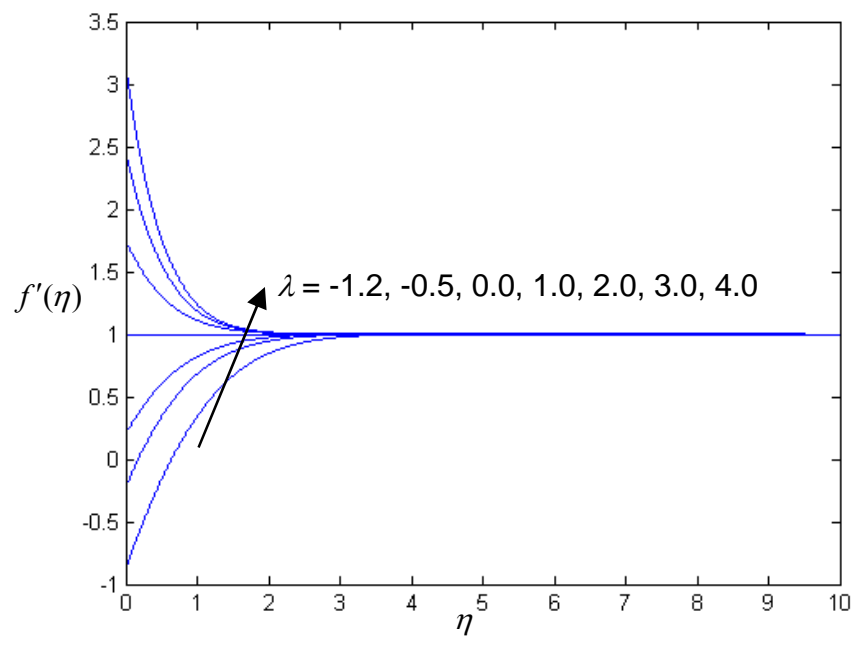

Fig. 1 Velocity profiles for some values of $\lambda$

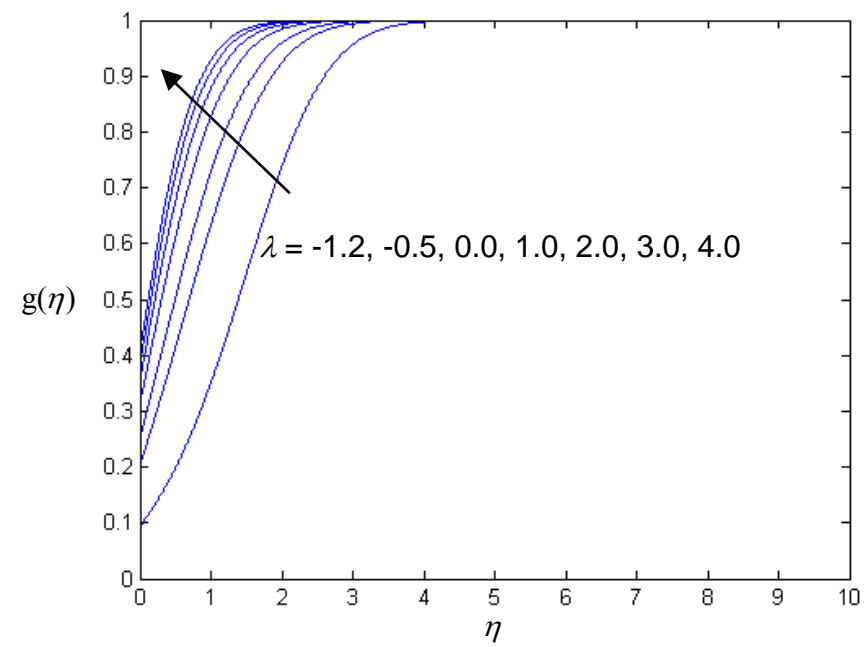

Fig. 2 Concentration profiles for some values of $\lambda$ 
The variations of the velocity and concentration profiles are plotted for different values of $M$ in Figures 3 and 4. In Fig.3, for $\lambda>0$ (stretching surface), the fluid velocity is becoming increasingly greater than the free stream. In this case the fluid velocity decreases with the value of $\eta$ and converges at unity as per the condition. For $\lambda<0$ (shrinking surface), the velocity decreases with the increase of magnetic parameter $M$ for opposing, assisting and steady state cases. That is because the application of a magnetic field in the y-direction to an electrically conducting fluid gives rise to a flow resistive force called the Lorentz force. The concentration profile in figure 4 , for $\lambda>0$ (stretching surface), the fluid concentration increases with increasing magnetic parameter $M$ and opposite case is observed for $\lambda<0$ (shrinking surface). All the curves are started from the origin and they increase nonlinearly with $\eta$ to follow ' $S$ ' shape and finally reach unity.

The solute velocity, however, increase with the permeability for stretching/shrinking parameters is observed from Fig.5. A concentration profile for different values of permeability parameter is shown in Fig.6. For $\lambda>0$ (stretching surface), the fluid concentration decreases with increasing the permeability parameter D and opposite case is observed for $\lambda<0$ (shrinking surface).

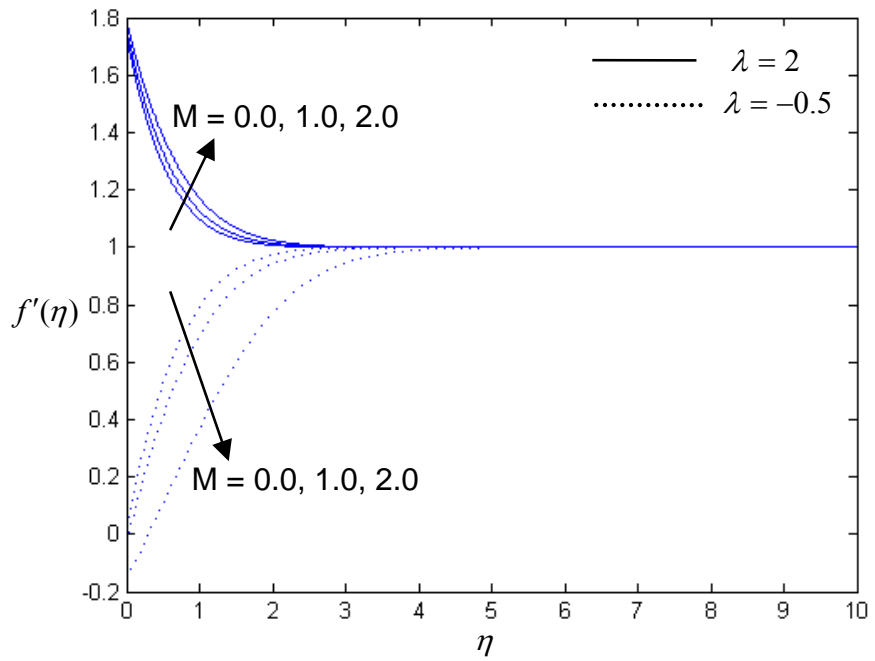

Fig. 3 Velocity profiles for some values of $M$

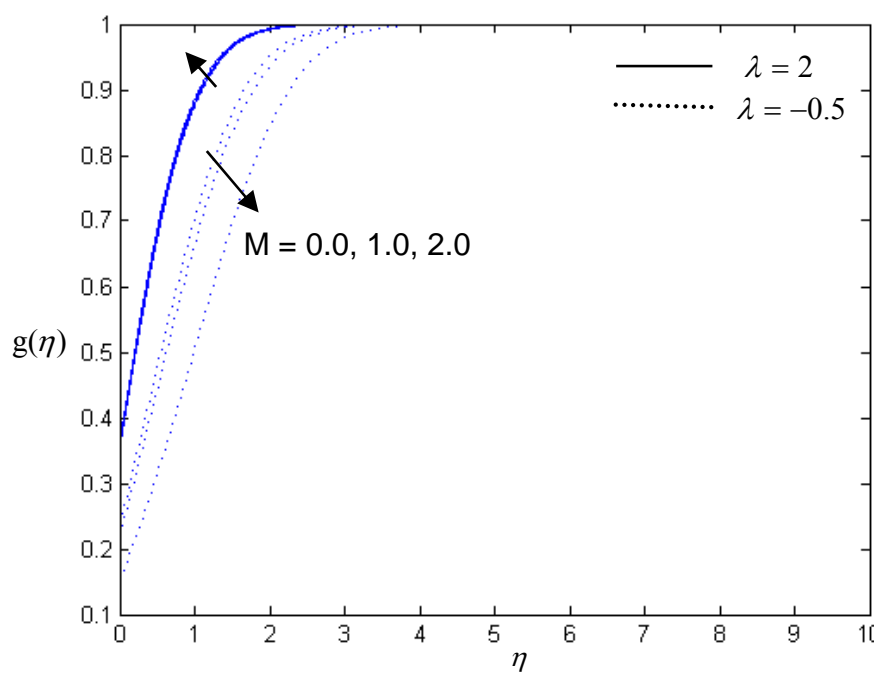

Fig. 4 Concentration profiles for some values of $M$

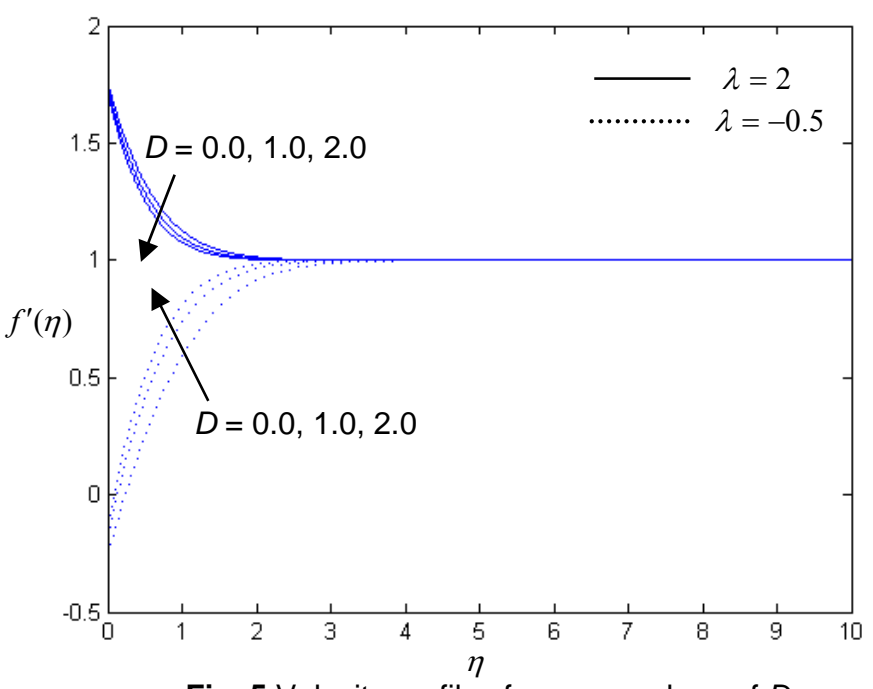

Fig. 5 Velocity profiles for some values of $D$

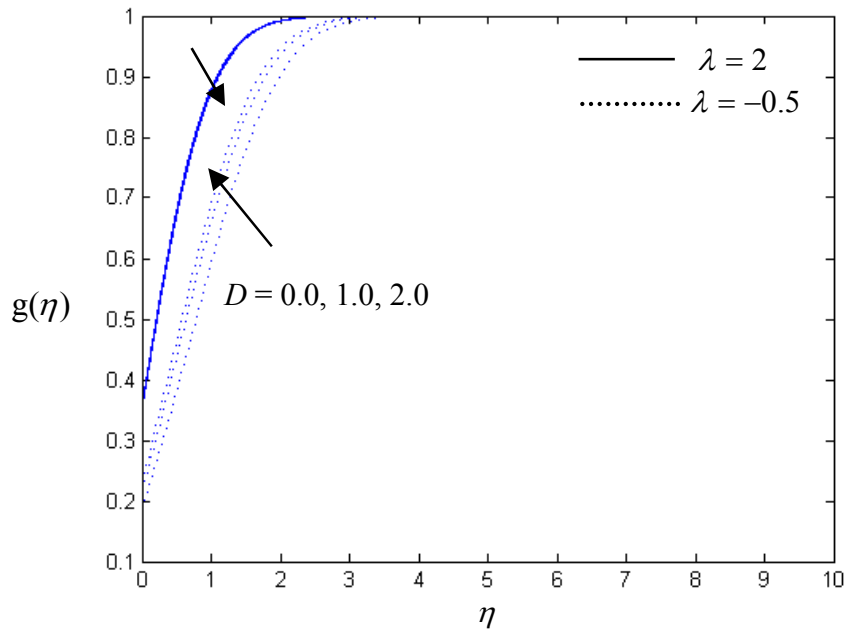

Fig. 6 Concentration profiles for some values of $D$

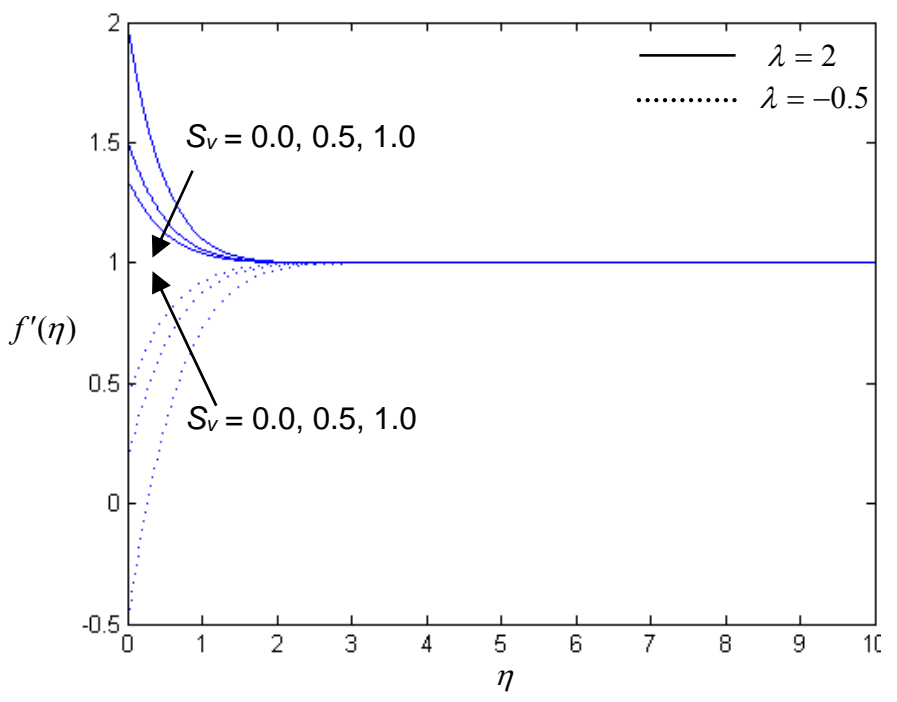

Fig. 7 Velocity profiles for some values of $S_{v}$ 
The variations of the velocity and concentration profiles are plotted for different values of slip parameter $\left(S_{v}\right)$ in Figures 7 and 8. In Fig.7, for $\lambda$ $>0$ the fluid velocity increases with the increase of slip parameter and an opposite effect is seen when $\lambda<0$. The increase in the slip parameter has the tendency to reduce the friction forces which reduces the fluid velocity. The fluid concentration enhances when $S_{v}$ enhances for $\lambda>0$ and decreases for $\lambda<0$ in Fig. 8 .

The effect of heterogeneous and homogeneous reactions on the concentration profile are separately shown through Figures 9 and 11 for stretching sheet and Figures 10 and 12 for shrinking sheet respectively. It is evident that the concentration boundary layer of the reactants is increasing with $\eta$ in both cases, and after a certain $\eta$ value, they all coincide, i.e., after a certain $\eta$ value, the homogeneous and heterogeneous reactions have no e ect on the concentration of the reactants. This critical value of $\eta\left(\eta_{\infty}\right)$ depends on the strength of the homogeneous reaction and increases with the value of $\mathrm{K}$, but it does not depend on the strength of the heterogeneous reaction. A similar phenomenon is observed for the second solution. The graphs for these condition solutions with $\mathrm{K}_{\mathrm{s}}=0.2$ and 1 coincide. It is observed that the first solution is more stable and converges more easily than the second solution.

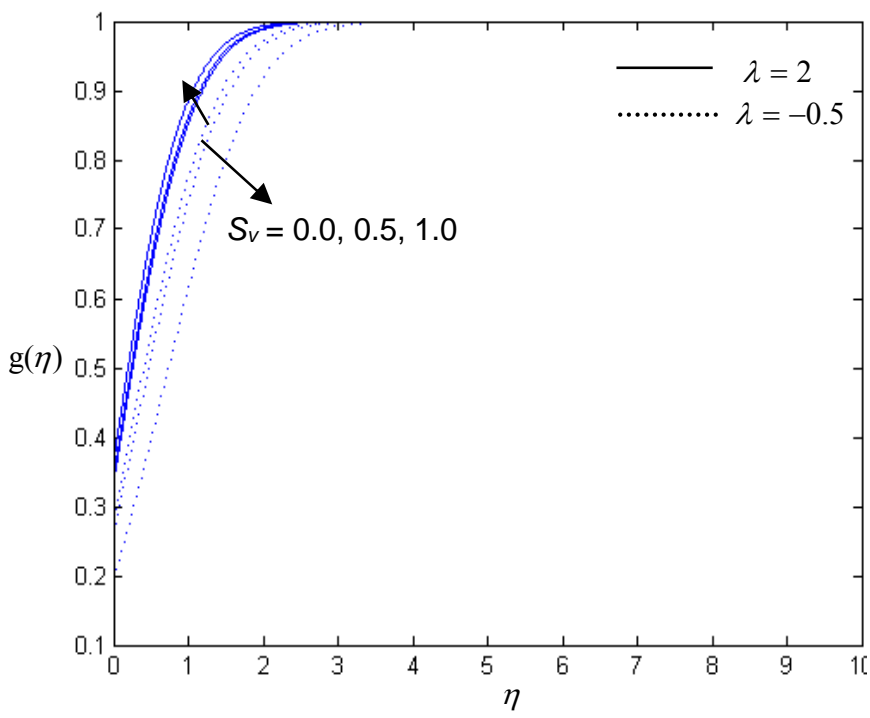

Fig. 8 Concentration profiles for some values of $S_{v}$

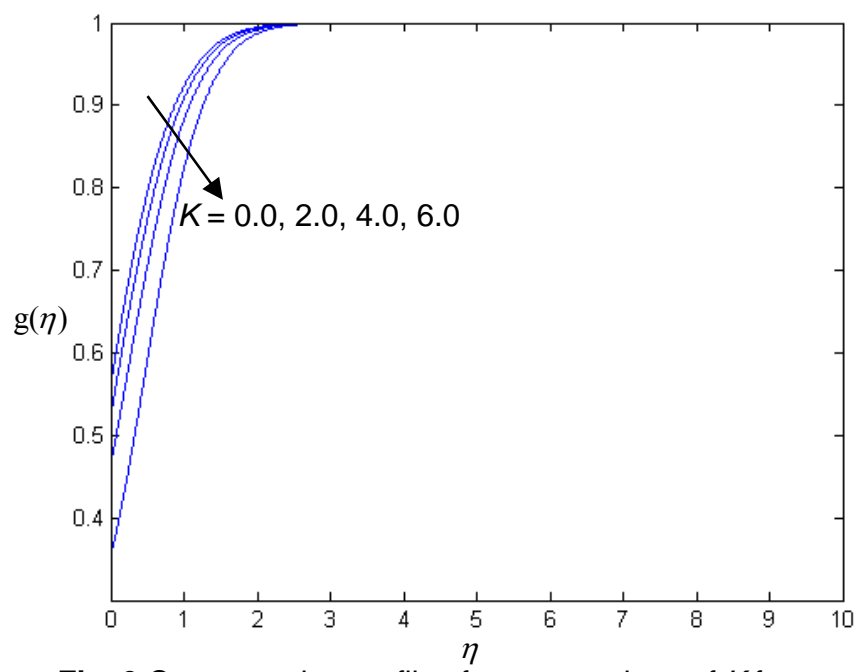

Fig. 9 Concentration profiles for some values of $K$ for stretching sheet

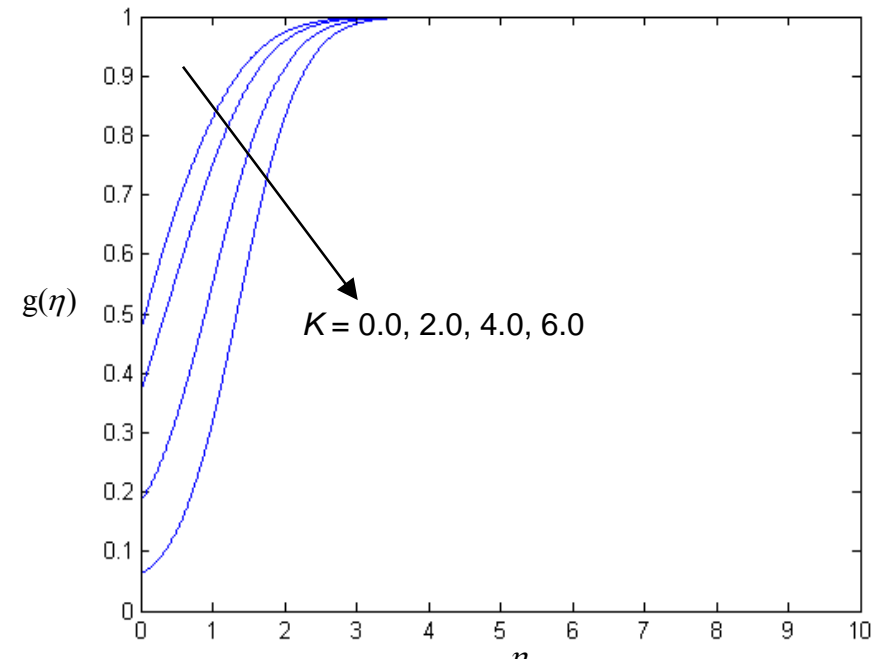

Fig. 10 Concentration profiles for some values of $K$ for shrinking sheet

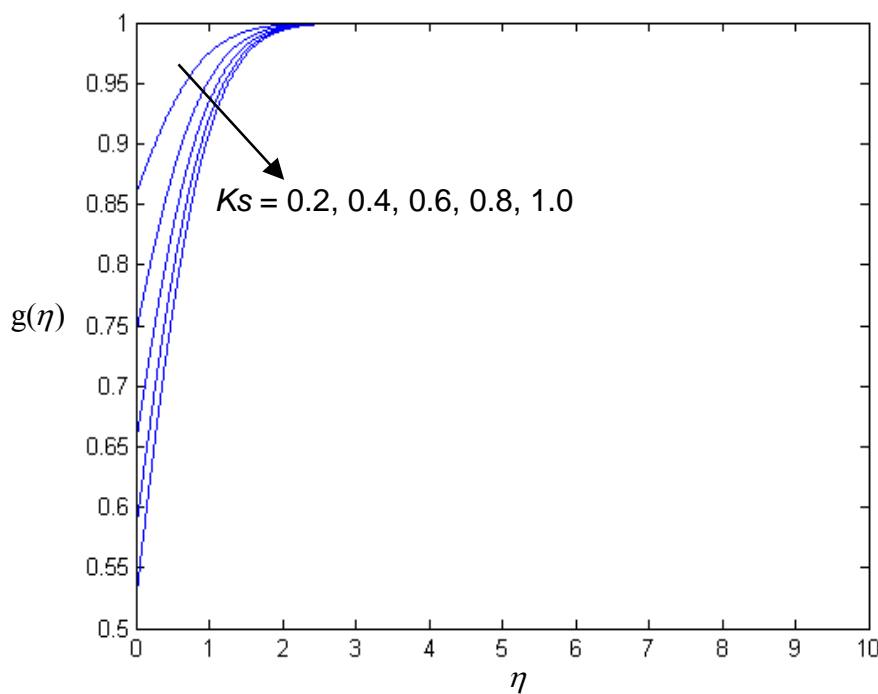

Fig. 11 Concentration profiles for some values of $K s$ for stretching sheet

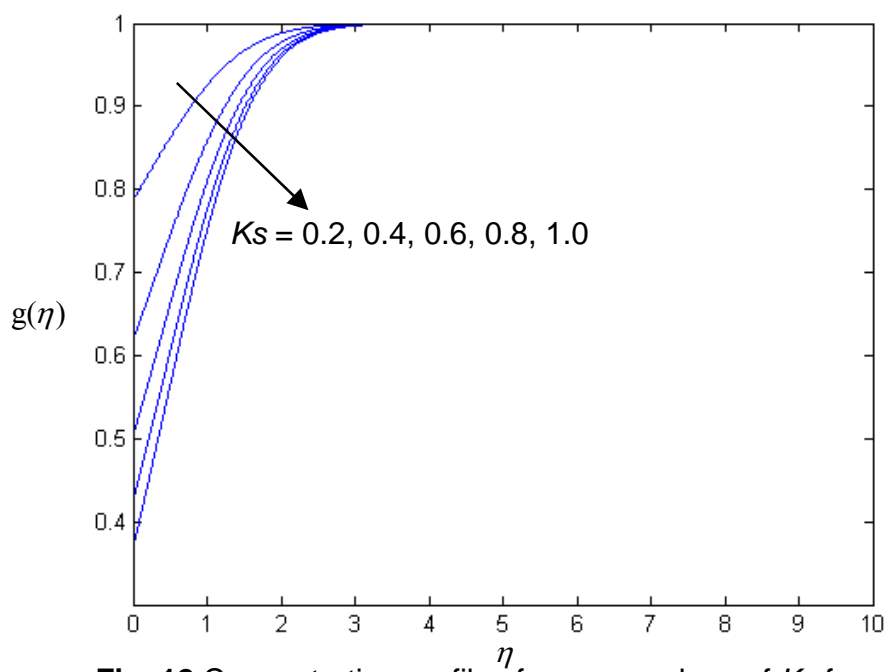

Fig. 12 Concentration profiles for some values of $K s$ for shrinking sheet 
The concentration of the reactants depends on the Schmid tnumber ( $\mathrm{Sc}$ ) and heterogeneous reaction parameter. The variation of the concentration with $\mathrm{K}$ for di erent values of the Schmidt number is shown in Figures 13 and 14. The Schmidt number is the ratio between a viscous di usion rate and a molecular di usion rate. For a fixed molecular di usion rate, with increase in Schmidt number, the viscous di usion rate increases, which helps to increase the concentration of the fluid for both stretching and shrinking sheet.

Figs.15 and 16 are aimed to shed light on the effect of suction ( $S=0$ represents impermeable, $S>0$ represents suction and $S<0$ represents the injection or blowing) on the velocity and concentration profiles. From these, we observed that the velocity decreases with an increase in the suction parameter whereas concentration increases for stretching sheet this is due to the fact that the heated fluid is pushed towards the wall where the buoyancy forces can act to retard the fluid due to high influence of viscosity. This effect acts to decrease the wall shear stress. The effect of the velocity and concentration profiles for different suction parameter $\mathrm{S}$ is shown in Figures 17 and 18. The velocity of the fluid increases due to increase of $\mathrm{S}$, and this leads to an increase in the solute concentration for shrinking sheet.

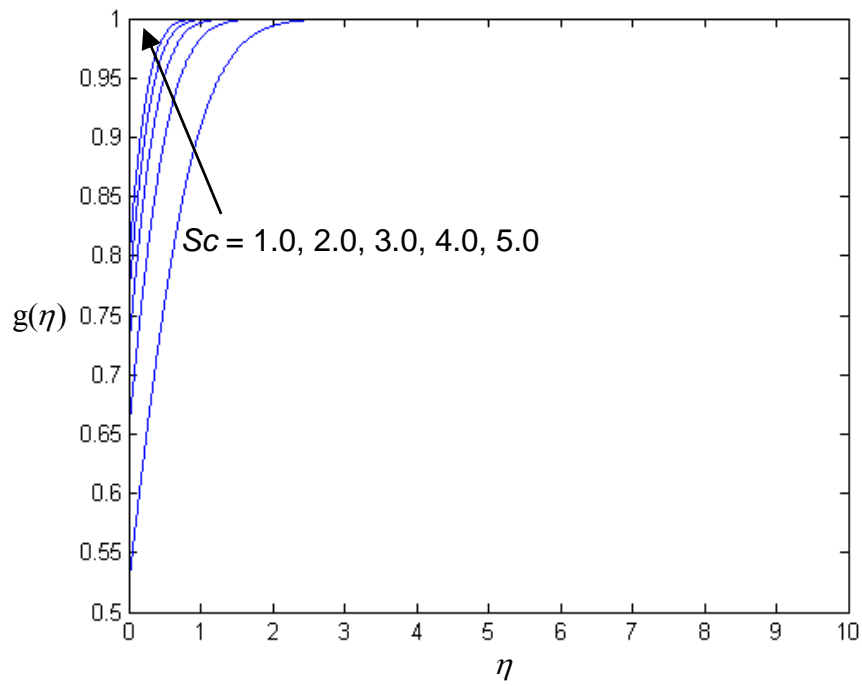

Fig. 13 Concentration profiles for some values of Sc for stretching sheet

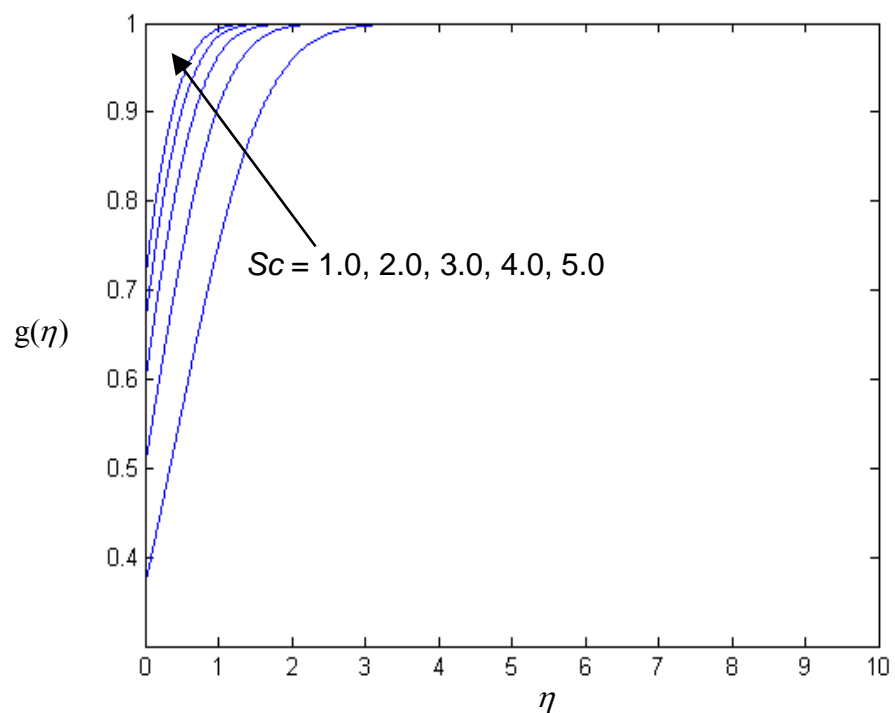

Fig. 14 Concentration profiles for some values of $S c$ for shrinking sheet

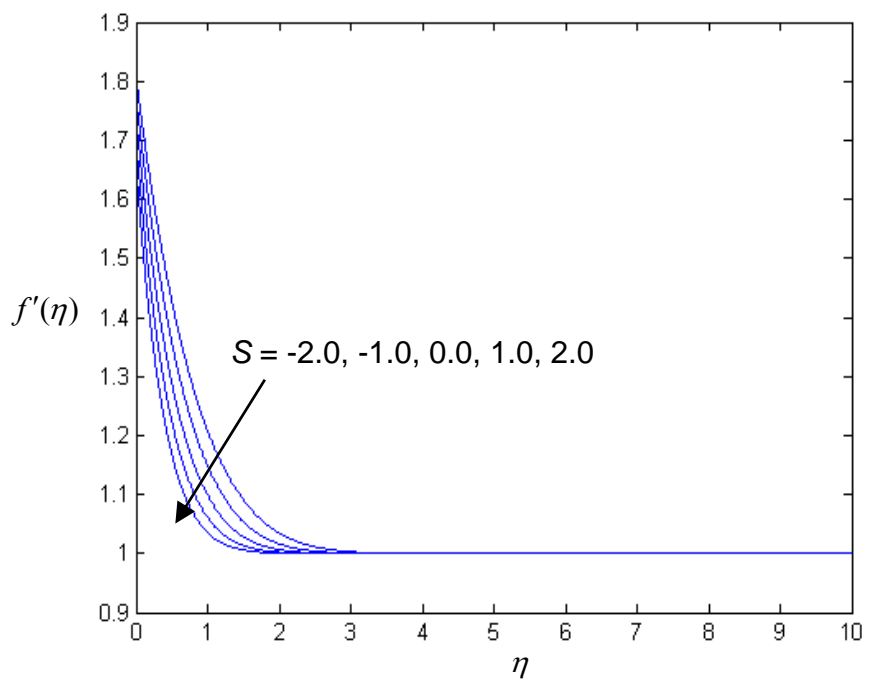

Fig. 15 Velocity profiles for some values of $S$ for stretching sheet

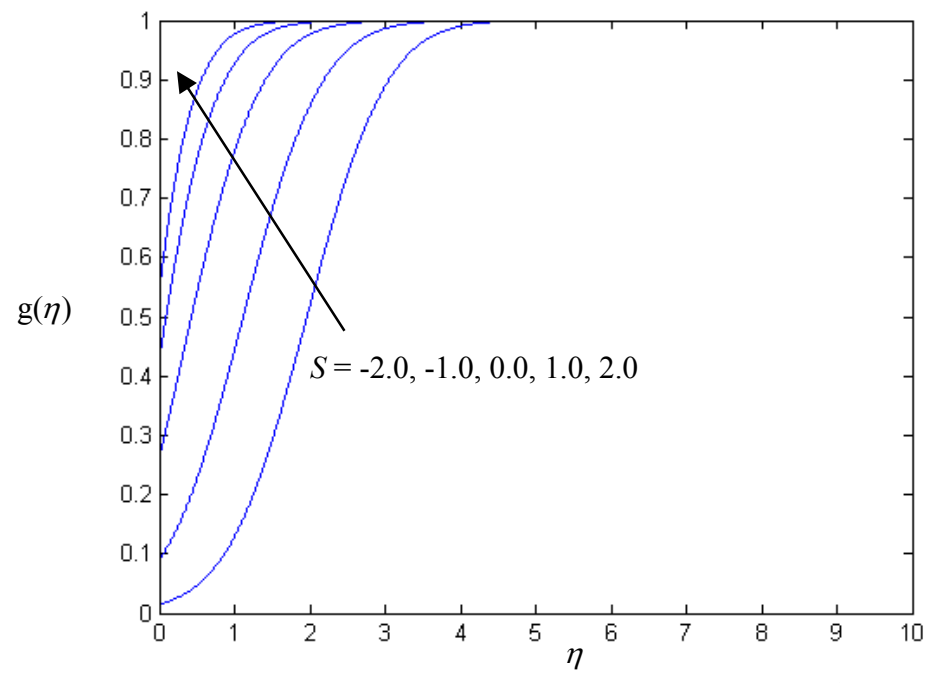

Fig. 16 Concentration profiles for some values of $S$ for stretching sheet

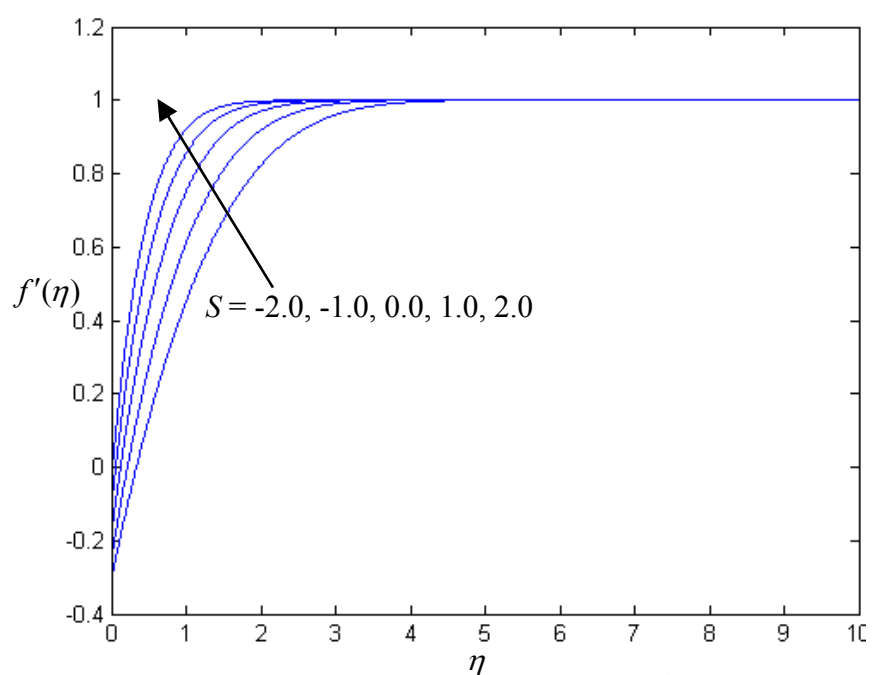

Fig. 17 Velocity profiles for some values of $S$ for shrinking sheet 


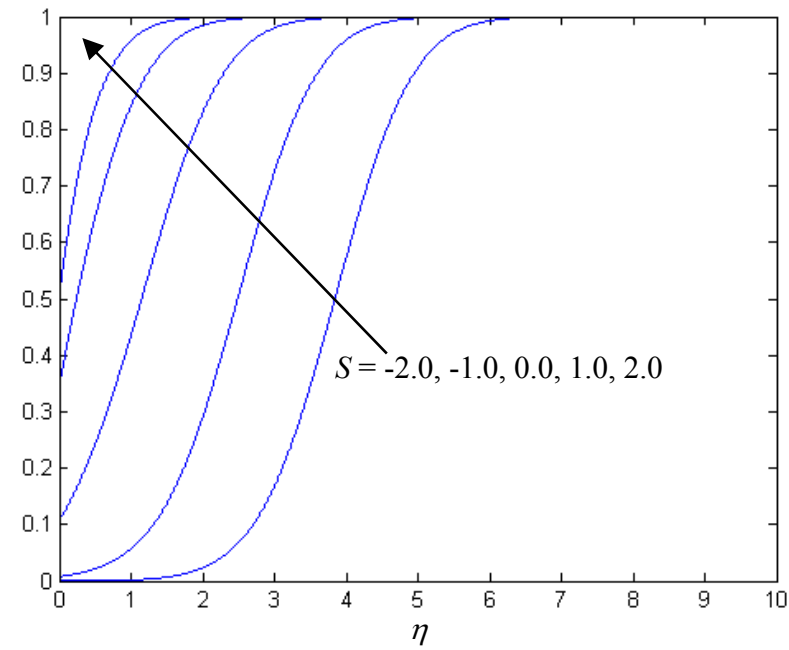

Fig. 18 Velocity profiles for some values of S for shrinking sheet

Values of the dimensionless skin friction coefficient $f^{\prime \prime}(0)$ for several values of $S$, suction parameter in the absence of micropolar parameter, magnetic parameter, permeability parameter and the slip velocity parameter are given in Table 1. The values reported by Katagiri (1971) using an iterative numerical quadratures and by Lok et al. (2007) using the Keller-box method were also included in this table. It is seen that the present results are in excellent agreement with both results obtained by Katagiri (1971), Lok et al. (2007) and Khan and Pop (2010). We notice that for an impermeable wall $(S=0)$ the values of $f^{\prime \prime}(0)$ reported by Hiemenz (1911) is $\mathrm{S}=1.233$.

Table 1 Comparison of $f^{\prime \prime}(0)$ for several values of $S$ in the absence of micropolar parameter, magnetic parameter, permeability parameter and the slip velocity parameter.

\begin{tabular}{|l|l|l|l|l|}
\hline$S$ & $\begin{array}{l}\text { Katagiri } \\
(1971)\end{array}$ & $\begin{array}{l}\text { Lok et al. } \\
(2007)\end{array}$ & $\begin{array}{l}\text { Khan and } \\
\text { Pop (2010) }\end{array}$ & Present \\
\hline-3 & 0.329456 & 0.3295 & 0.32945 & 0.32945 \\
\hline-2 & 0.47581 & 0.4759 & 0.47581 & 0.47581 \\
\hline-1 & 0.756574 & 0.7567 & 0.75657 & 0.75657 \\
\hline 0 & 1.232588 & 1.2327 & 1.23259 & 1.23258 \\
\hline 1 & 1.889303 & 1.8895 & 1.88931 & 1.88931 \\
\hline 2 & 2.670006 & 2.6703 & 2.67006 & 2.67005 \\
\hline 3 & 3.526497 & 3.5268 & 3.52664 & 3.52664 \\
\hline 4 & 4.428673 & 4.4291 & 4.42895 & 4.42895 \\
\hline
\end{tabular}

Table 2, present the excellent correlation between previous literatures [Wang (2008), Ishak et al. (2010), Rosali et al. (2012)] and the present study of the comparison of $\mathrm{f}^{\prime \prime}(0)$ for several values of $\lambda$ in the absence of micropolar parameter, magnetic parameter, permeability parameter, suction parameter and the slip velocity parameter for a stretching sheet. This investigation confirms that the existence and uniqueness of solution depends on the stretching/shrinking sheet parameter. $\lambda=0$ Represents the forced convection flow towards the stagnation point on a static surface. It is clear that the skin friction is a decreasing function of $\lambda$. All values of the skin friction coefficient are positive for $\lambda<1$, while they are negative when $\lambda>1$. Physically, the negative values of the skin friction coefficient correspond to the surface exerting a drag force on the fluid and the opposite sign implies the inverse phenomenon. The skin friction coefficient is zero when $\lambda=1$ regardless of the values of other parameters. This is because for $\lambda=1$, there is no shear stress at the surface as the surface and fluid move with the same velocity.

Table 2 Comparison of $f^{\prime \prime}(0)$ for several values of $\lambda$ in the absence of micropolar parameter, magnetic parameter, permeability parameter, suction parameter and the slip velocity parameter

\begin{tabular}{|l|l|l|l|l|l|l|}
\hline$\lambda$ & \multirow{2}{*}{$\begin{array}{l}\text { Wang } \\
\text { (2008) }\end{array}$} & $\begin{array}{l}\text { Ishak et al } \\
(2010)\end{array}$ & \multicolumn{2}{|c|}{ Rosali et al. (2012) } & \multicolumn{2}{|c|}{ Present } \\
\cline { 4 - 7 } & & & $\begin{array}{l}K_{p}=0, S \\
=0, D= \\
0\end{array}$ & $\begin{array}{l}K_{p}=1, S \\
=0.8, D \\
=0.5\end{array}$ & $\begin{array}{l}K_{p}=0, S \\
=0, D= \\
0\end{array}$ & $\begin{array}{l}K_{p}=1, S \\
=0.8, D \\
=0.5\end{array}$ \\
\hline 0.0 & 1.232588 & 1.232588 & 1.232588 & 1.476217 & 1.232588 & 1.476217 \\
\hline 0.1 & 1.14656 & 1.146561 & 1.146561 & 1.353345 & 1.146561 & 1.353345 \\
\hline 0.2 & 1.05113 & 1.051130 & 1.05113 & 1.224482 & 1.05113 & 1.224482 \\
\hline 0.3 & - & - & 0.946816 & 1.089841 & 0.946816 & 1.089841 \\
\hline 0.4 & - & - & 0.834072 & 0.949614 & 0.834072 & 0.949614 \\
\hline 0.5 & 0.71330 & 0.713295 & 0.713295 & 0.803979 & 0.713295 & 0.803979 \\
\hline 1.0 & 0.00000 & 0.00000 & 0.00000 & 0.00000 & 0.00000 & 0.00000 \\
\hline 2.0 & -1.88731 & -1.887307 & -1.887307 & -1.941163 & -1.887307 & -1.941163 \\
\hline 3.0 & - & -4.276541 & -4.276541 & -4.260253 & -4.276541 & -4.260253 \\
\hline 4.0 & - & -7.086378 & -7.086378 & -6.904439 & -7.086378 & -6.904439 \\
\hline 5.0 & -10.26475 & -10.26479 & -10.26479 & -9.837608 & -10.26479 & -9.837608 \\
\hline
\end{tabular}

From Table 3 it is clear that as the micro polar parameter or the magnetic parameter increases both $f^{\prime \prime}(0)$ and $g(0)$ increases. As the diffusion coefficient and Schmidt number increases both $f^{\prime \prime}(0)$ and $g(0)$ remains constant. As $\lambda$ increases a tremendous decrease is seen in $f^{\prime \prime}(0)$, a reverse effect is seen when a slip parameter increases. Homogeneous and heterogeneous reactions do not effect $f^{\prime \prime}(0)$ and $\mathrm{g}(0)$. Here $n=0$ represents the strong concentration and $n=1$ represents the turbulent boundary layer flow. The case $n=1 / 2$ indicates the vanishing of the anti-symmetrical part of the stress tensor and denotes weak concentration which is the case considered in the present study.

Table 3 The values of skin friction coefficient and dimensionless concentration for various values of $K p, M, D, S c, \lambda$, and $S_{v} K s, K$ and $n$.

\begin{tabular}{|l|l|l|l|l|l|l|l|l|l|l|l|}
\hline$K_{p}$ & $M$ & $D$ & $S c$ & $S$ & $\lambda$ & $S_{v}$ & $K s$ & $K$ & $n$ & $f^{\prime \prime}(0)$ & $g(0)$ \\
\hline 1.0 & 0.5 & 0.5 & 1.0 & 0.5 & 2.0 & 0.2 & 1.0 & 1.0 & 0.5 & -1.2533 & 0.5472 \\
\hline 2.0 & 0.5 & 0.5 & 1.0 & 0.5 & 2.0 & 0.2 & 1.0 & 1.0 & 0.5 & -1.1128 & 0.5509 \\
\hline 1.0 & 1.0 & 0.5 & 1.0 & 0.5 & 2.0 & 0.2 & 1.0 & 1.0 & 0.5 & -1.1947 & 0.5487 \\
\hline 1.0 & 0.5 & 1.0 & 1.0 & 0.5 & 2.0 & 0.2 & 1.0 & 1.0 & 0.5 & -1.3064 & 0.5459 \\
\hline 1.0 & 0.5 & 0.5 & 2.0 & 0.5 & 2.0 & 0.2 & 1.0 & 1.0 & 0.5 & -1.2533 & 0.6653 \\
\hline 1.0 & 0.5 & 0.5 & 1.0 & 1.0 & 2.0 & 0.2 & 1.0 & 1.0 & 0.5 & -1.3623 & 0.6153 \\
\hline 1.0 & 0.5 & 0.5 & 1.0 & 0.5 & 3.0 & 0.2 & 1.0 & 1.0 & 0.5 & -2.6734 & 0.5738 \\
\hline 1.0 & 0.5 & 0.5 & 1.0 & 0.5 & 2.0 & 1.0 & 1.0 & 1.0 & 0.5 & -0.6138 & 0.5308 \\
\hline 1.0 & 0.5 & 0.5 & 1.0 & 0.5 & 2.0 & 0.2 & 2.0 & 1.0 & 0.5 & -1.2533 & 0.3747 \\
\hline 1.0 & 0.5 & 0.5 & 1.0 & 0.5 & 2.0 & 0.2 & 1.0 & 2.0 & 0.5 & -1.2533 & 0.5274 \\
\hline 1.0 & 0.5 & 0.5 & 1.0 & 0.5 & 2.0 & 0.2 & 1.0 & 1.0 & 1.0 & -1.4587 & 0.5420 \\
\hline
\end{tabular}

\section{CONCLUSIONS}

The present analysis investigates the effect of the homogeneous and heterogeneous chemical reaction and slip velocity on MHD stagnation flow of a micropolar fluid flow through a permeable stretching/shrinking sheet embedded in a porous medium. The momentum and concentration equations were transformed into a set of 
coupled nonlinear differential equations using similarity transformations and solved numerically by Matlab bvp4c package. We discussed the effects of the governing parameters on the fluid flow and concentration characteristics. A new feature that emerges from our results It is found that these solutions terminate at $\lambda=0$ with values given in Table 1 . The concentration profiles $\mathrm{g}(\mathrm{g})$ appear to be similar in shape for different values of $\lambda, \mathrm{K}$ and $\mathrm{Ks}$. There is an excellent correlation between previous literatures and the present study.

\section{REFERENCES}

Abo-Eldahab E and MGhonaim A. F., 2003," Convective Heat Transfer in an Electrically Conducting Micropolar Fluid at a Stretching Surface with Uniform Free Stream", Applied Mathematics and Computation 137, 323-336.

Ahmadi G., 1976," Self-Similar Solution of Incompressible Micropolar Boundary Layer Flow Over a Semi-Infinite Plate", Int J Eng. Sci, 14, 639-646.

Ahmad A. F., Hussain S., Alanbari A.M. and Alharbi R. S., 2015," MHD Flow and Heat Transfer Through a Porous Medium Over a Stretching/Shrinking Surface with Suction", Sci.Int.(Lahore), 27(2), 931-935, Issn 1013-5316; Coden: Sinte 8.

Alireza R., Farzaneh-Gord M., Varedi S. R and Ganji D.D., 2013," Analytical Solution for Magnetohydrodynamic Stagnation Point Flow and Heat Transfer over a Permeable Stretching Sheet with Chemical Reactionl", Journal of Theoretical and Applied Mechanics, 51(3), 675686, Warsaw.

Ariman T., Turk MA, Sylvester ND., 1974 a," On Steady and Pulsatile Flow of Blood", J.Appl. Mech, 41, 1-7.

http://dx.doi.org/10.1115/1.3423224

Ariman T., Turk M.A and Sylvester. ND., 1974 b" Micro Continuum Fluid Mechanics - A Review", Int. J. Eng. Sci. 12, 273-293.

Bakr A.A., (2011) "Effects of Chemical Reaction on MHD Free Convection and Mass Transfer Flow of a Micropolar Fluid with Oscillatory Plate Velocity and Constant Heat Source in a Rotating Frame of Reference", Commun. Nonlinear Sci. Numer. Simul., 16, 698710.

http://dx.doi.org/10.1016/j.cnsns.2010.04.040

Beaver G. S. and Joseph DD., 1967," Boundary Conditions at a Naturally Permeable Wall. J. Fluid Mech", 30, 197 - 207.

http://dx.doi.org/10.1017/S0022112067001375

Chambre PL., Acrivos A., 1956," On Chemical Surfaces Reactions in Laminar Boundary Layer Flows", J. Appl. Phys., 1322-1328.

http://dx.doi.org/10.1063/1.1722258

Chaudhary MA and Merkin JH., 1995 a," A Simple Isothermal Model for Homogeneous-Heterogenous Reactions in Boundary-Layer Flow I". Equal diffusivities. Fluid Dyn. Res "16, 311-333.

http://dx.doi.org/10.1016/0169-5983(95)00015-6

Chaudhary MA and Merkin JH., 1995 b, "A Simple Isothermal Model for Homogeneous-Heterogenous Reactions in Boundary-Layer Flow II," Unequal diffusivities. Fluid Dyn. Res. 16, 335-359.

Chen C. K and. Char M. I., 1988, "Heat Transfer of a Continuous Stretching Surface with Suction or Blowing," Journal of Mathematical Analysis and Applications 135, 568-580.

http://dx.doi.org/10.1016/0022-247X(88)90172-2
Chiam T.C., 1994, "Stagnation-Point Flow towards a Stretching Plate," J. Phys. Soc. Jpn, 63, 2443-2444.

Crane LJ., 1970," Flow Past a Stretching Plate, Zeitschrift Fur Angewandte Mathematik Und Physik", 21, 645-647.

Dutta B. K., Roy P., and Gupta A. S., 1985," Temperature Field in The Flow Over a Stretching Sheet with Uniform Heat Flux," International Communications in Heat and Mass Transfer, 12, 89-94. http://dx.doi.org/10.1016/0735-1933(85)90010-7

Eldabe N. T., Mahmoud E.M., Ouaf., 2006, “Chebyshev Finite Difference Method for Heat and Mass Transfer in a Hydromagnetic Flow of a Micropolar Fluid Past a Stretching Surface with Ohmic Heating and Viscous Dissipation", Applied Mathematics and Computation 177, 561-571.

http://dx.doi.org/10.1016/j.amc.2005.07.071

Eringen AC., 1966," Theory of Micropolar Fluids". J. Math. Mech, 16, $1-18$.

Eringen AC., 1976, Non-Local Polar Field Theory. Academic Press, New York.

Gupta PS., and Gupta AS., 1977,'Heat and Mass Transfer on a Stretching Sheet with Suction or Blowing ", Canadian Journal of chemical Engineering, 55, 744-746.

http://dx.doi.org/10.1002/cjce.5450550619

Guram GS, and Smith AC.,1980,'Stagnation Flows of Micropolar Fluids with Strong and Weak Interactions", Comput. Math.Appl, 6, $213-$ 233.

Haliza Rosali, Anuar Ishak, Ioan Pop., 2012, " A Micropolar Fluid Flow Towards a Permeable Stretching/Shrinking Sheet in a Porous Medium," International Communications in Heat and Mass Transfer, 39, 826-829.

http://dx.doi.org/10.1016/j.icheatmasstransfer.2012.04.008

Hayat T., Javed T., and Abbas Z., 2009,'MHD Flow of a Micropolar Fluid Near a Stagnation-Point Towards a Non-Linear Stretching Surface," Nonlinear Anal: Real World Appl, 10, 1514-1526.

http://dx.doi.org/10.1016/j.nonrwa.2008.01.019

Hayat T., Abbas Z., and Sajid M., 2009," MHD Stagnation Point Flow of An Upper Convected Maxwell Fluid Over a Stretching Sheet," Chaos Solitons and Fractals, 39, 840-848.

http://dx.doi.org/10.1016/j.chaos.2007.01.067

Hayat T., Farooq S., Ahmad B., Alsaedi A., 2016, "HomogeneousHeterogeneous Reactions and Heat Source/Sink Effects in MHD Peristaltic Flow of Micropolar Fluid with Newtonian Heating in A Curved Channel, Journal of Molecular Liquids 223, 469-488. http://dx.doi.org/10.1016/j.molliq.2016.08.067

Hayat T., Zakir Hussain, Taseer Muhammad, 2016, “Ahmed Alsaedi, Effects of Homogeneous and Heterogeneous Reactions in Flow of Nanofluids Over a Nonlinear Stretching Surface with Variable Surface Thickness," Journal of Molecular Liquids, 221, 1121-1127. http://dx.doi.org/10.1016/j.molliq.2016.06.083

Hayat T., Zakir Hussain, Alsaedi A., Ahmad B., 2016, "HeterogeneousHomogeneous Reactions and Melting Heat Transfer Effects in Flow with Carbon Nanotubes," Journal of Molecular Liquids 220, 200-207. http://dx.doi.org/10.1016/j.molliq.2016.04.012 
Hayat T., Zakir Hussain, Alsaedi A., Asghar S., 2016 "Carbon Nanotubes Effects in The Stagnation Point Flow Towards a Nonlinear Stretching Sheet with Variable Thickness," Advanced Powder Technology $\mathrm{xxx} \quad \mathrm{xxx}-\mathrm{xxx}$ (Article in Press) http://dx.doi.org/10.1016/j.apt.2016.06.001

Hayat T., Zakir Hussain, Alsaedi A., Farooq M., 2016, "Magnetohydrodynamic Flow by a Stretching Cylinder with Newtonian Heating and Homogeneous-Heterogeneous Reactions," Plops ONE 11, $1-23$.

http://dx.doi.org/10.1371/journal.pone.0156955

Hayat T., Zakir Hussain, Farooq M., Alsaedi A., 2016, "Effects of Homogeneous and Heterogeneous Reactions and Melting Heat in the Viscoelastic Fluid Flow," Journal of Molecular Liquids 215, 749-755. http://dx.doi.org/10.1016/j.molliq.2015.12.109

Hayat T., Zakir Hussain,M. Farooq, Alsaedi, A., 2016, "Homogeneous and Heterogeneous Reactions Effects in Flow With Joule Heating and Viscous Dissipation," Journal of Mechanics, 1-10.

http://dx.doi.org/10.1017/jmech.2016.71

Tasawar Hayat, Sumaira Qayyum, Maria Imtiaz, Faris Alzahrani and Ahmed Alsaedi, 2016, "Partial Slip Effect in Flow of Magnetite-Fe3O4 Nanoparticles Between Rotating Stretchable Disks," Journal of Magnetism and Magnetic Materials, $413 \quad 39$. http://dx.doi.org/10.1016/j.jmmm.2016.04.025

Tasawar Hayat, Maria Imtiaz and Ahmed Alsaedi, 2015, "Impact of Magnetohydrodynamics In Bidirectional Flow of Nanofluid Subject to Second Order Slip Velocity and Homogeneous-Heterogeneous Reactions," Journal of Magnetism and Magnetic Materials, 395294. http://dx.doi.org/10.1016/j.jmmm.2015.07.092

Tasawar Hayat, Maria Imtiaz, Ahmed Alsaedi, Marwan A. Kutbi, 2015, "MHD Three-Dimensional Flow of Nanofluid With Velocity Slip and Nonlinear Thermal Radiation," Journal of Magnetism and Magnetic Materials, 396 31-37.

http://dx.doi.org/10.1016/j.jmmm.2015.07.091

Hayat T., Maryam Shafique, Anum Tanveer, Alsaedi, A., 2016, "Magnetohydrodynamic Effects on Peristaltic Flow of Hyperbolic Tangent Nanofluid With Slip Conditions and Joule Heating in an Inclined Channel," International Journal of Heat and Mass Transfer 102, 54-63.

http://dx.doi.org/10.1016/j.ijheatmasstransfer.2016.05.105

Hiemenz K.,1911,"Die Grenzschicht an Einem in Den Gleichformingen Flussigkeits-Strom Einge-Tauchten Graden Kreiszylinder," Dingler's Poly.J., 326, 321-324.

Hudimoto B., Tokuoka T., 1969, "Two-Dimensional Shear Flows of Linear Micropolar Fluids", Int. J. Eng. Sci. 7, 515-522.

http://dx.doi.org/10.1016/0020-7225(69)90036-6

Misra JC., and Ghosh S.K., 1997," A Mathematical Model for The Study of Blood Flow Through a Channel with Permeable Walls". Acta Mech., 122, 137 - 153.

Motsa1 SS., and Shateyi S., 2012, "The Effects of Chemical Reaction, Hall, and Ion-Slip Currents on MHD Micropolar Fluid Flow with Thermal Diffusivity Using a Novel Numerical Technique", Journal of Applied Mathematics, 2012, Article ID 689015, 30 pages.

http://dx.doi.org/10.1155/2012/689015
Ishak A., Lok YY., Pop I., 2010," Stagnation Point Flow over a Shrinking Sheet in a Micropolar Fluid". Chem. Eng. Commun., 197, 1417-1427.

http://dx.doi.org/10.1080/00986441003626169

Maryiam Javed, Hayat T., Mustafa M., Ahmad B., 2016, "Velocity and Thermal Slip Effects on Peristaltic Motion of Walters-B Fluid," International Journal of Heat and Mass Transfer 96, 210-217 http://dx.doi.org/10.1016/j.ijheatmasstransfer.2015.12.029

Katagiri M.,1971,'Unsteady Boundary Layer Near the Forward Stagnation Point with Uniform Suction or Injection". J Phys Soc Japan, 31,935-9.

Khan WA., Pop I., 2012,'Effects of Homogeneous-Heterogeneous Reactions on The Viscoelastic Fluid Toward a Stretching Sheet," J. Heat Transfer, 134, 064506.

Khan WA., and Pop I., 2010,'Flow Near the Two-Dimensional Stagnation Point on An Infinite Permeable Wall with A HomogeneousHeterogeneous Reaction", Commun Nonlinear Sci. Numer. Simul., 15, 3435-3443.

http://dx.doi.org/10.1016/j.cnsnc.2009.12.022

Krishnendu Bhattacharyya, 2013,'Boundary Layer Stagnation-Point Flow of Casson Fluid and Heat Transfer Towards a Shrinking/Stretching Sheet", Frontiers in Heat and Mass Transfer (FHMT), 4, 1-9.

http://dx.doi.org/10.5098/hmt.v4.2.3003

Lockwood F., Benchaita F., Friberg S., 1987," Study of Lyotropic Liquid Crystal in Viscometric Flow and Elasto Hydrodynamics Contact," Tribol. Trans, 30, 539-548.

http://dx.doi.org/10.1080/05698198708981790

Lok YY., Amin N., Pop I., 2007," Unsteady Boundary Layer Flow of a Micropolar Fluid Near a Stagnation Point with Uniform Suction or Injection", J. Teknologi (University Teknologi Malaysia), 46, 15-32.

Mahmoud M., and Waheed S.,2010," Effects of Slip and Heat Generation/Absorption on MHD Mixed Convection Flow of a Micropolar Fluid Over a Heated Stretching Surface", Mathematical Problems in Engineering, 2010, Article ID 579162, 20.

http://dx.doi.org/10.1155/2010/579162

Mahapatra TR., and Gupta A.S.,2001,'Magnetohydrodynamic Stagnation-Point Flow towards a Stretching Sheet. Acta Mech"152, 191-196.

http://dx.doi.org/10.1007/BF01176953

Merkin JH., 1996, "A Model for Isothermal HomogeneousHeterogeneous Reactions in Boundary-Layer Flow", Math. Comput. Model. 24, 125-136.

http://doi.org/10.1016/0895-7177(96)00145-8

Nazar R., Amin N., Filip D., Pop I., 2004," Stagnation Point Flow of a Micropolar Fluid Towards a Stretching Sheet", International Journal of Non-Linear Mechanics, 39, 1227-1235.

http://doi.org/10.1016/j.ijnonlinmec.2003.08.007

Peddieson J.,1972," An Application of the Micropolar Fluid Model to the Calculation of Turbulent Shear Flow", International Journal of Engineering Science, 10, 23-32.

Ramzan M., Farooq M., Hayat T., Jae Dong Chung., 2016, "Radiative and Joule Heating Effects in The MHD Flow of a Micropolar Fluid 
with Partial Slip and Convective Boundary Condition," Journal of Molecular Liquids 221, 394.

http://dx.doi.org/10.1016/j.molliq.2016.05.091

Rosali H., Ishak A., Pop I., 2012," Micropolar Fluid Flow Towards a Stretching/Shrinking Sheet in A Porous Medium with Suction", Int. Commun. Heat Mass Transf., 39, 826-829.

http://dx.doi.org/10.1016/j.icheatmasstransfer.2012.04.008

Sachin Shaw, Peri K Kameswaran and Precious Sibanda., 2013," Homogeneous- Heterogeneous Reactions in Micropolar Fluid from A Permeable Stretching or Shrinking Sheet in A Porous Medium", Boundary Value Problems 2013:77.

Saffman P G., 1971," On the Boundary Conditions at The Surface of a Porous Medium", Stud. Appl. Math., 1, 93 - 101.

Sajid M., Abbas Z., Hayat T., 2009, "Homotopy Analysis for Boundary Layer Flow of a Micropolar Fluid Through a Porous Channel," Applied Mathematical Modelling 33, 4120-4125.

http://dx.doi.org/10.1016/j.apm.2009.02.006

Sajid M., Ali N., Hayat T., 2009, “On Exact Solutions for Thin Film Flows of a Micropolar Fluid," Communications in Nonlinear Science and Numerical Simulation 14,451-461.

http://dx.doi.org/10.1016/j.cnsns.2007.09.003

Shampine LF., and Kierzenka J.,2000," Solving Boundary Value Problems for Ordinary Differential Equations in MATLAB with bvp4c ", Tutorial Notes.
Shehzad S.A., Abbasi F.M., Hayat T., Alsaadi F., Mousa G., 2015, "Peristalsis in A Curved Channel with Slip Condition and Radial Magnetic Field," International Journal of Heat and Mass Transfer 91, 562-569.

http://dx.doi.org/10.1016/j.ijheatmasstransfer.2015.07.076

Hayat T., Zakir Hussain, Taseer Muhammad, Ahmed Alsaedi, 2016, "Effects of Homogeneous and Heterogeneous Reactions in Flow of Nanofluids Over a Nonlinear Stretching Surface with Variable Surface Thickness," Journal of Molecular Liquids, 221 (2016) 1121-1127. http://dx.doi.org/10.1016/j.molliq.2016.06.083

Wang CY., 2008" Stagnation Flow Towards a Shrinking Sheet", Int. J. Non-Linear Mech., 43, 377-382.

Yongqi Wang, Nasir Ali, 2011, Tasawar Hayat, Martin Oberlack, "Peristaltic Motion of a Magnetohydrodynamic Micropolar Fluid in a Tube," Applied Mathematical Modelling, 35, 3737-3750. http://dx.doi.org/10.1016/j.apm.2011.02.030

Yacos N.A., Ishak A., Pop I., 2011, "Melting Heat Transfer in Boundary Layer Stagnation-Point Flow Towards a Stretching/Shrinking Sheet in A Micropolar Fluid", Comput. Fluids, 47 (1) 16-21. http://dx.doi.org/10.1016/j.compfluid.2011.01.040

Ziabakhsh Z., Domairry G., Bararnia H., Babazadeh H., 2010," Analytical Solution of Flow and Diffusion of Chemically Reactive Species Over a Nonlinearly Stretching Sheet Immersed in A Porous Medium", J. Taiwan Inst. Chem. Eng., 41, 22-28. http://dx.doi.org/10.1016/j.jtice.2009.04.011 